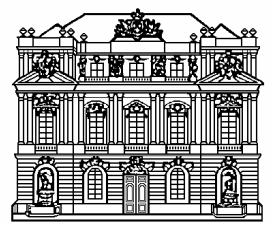

des theoretisch wissenschaftlichen Teiles

\title{
3 Problemstellung und Ansatz
}

\author{
Projektwerber und Projektleitung: \\ INSTITUT FÜR STRASSEN- UND VERKEHRSWESEN (ISV) \\ Technische Universität Graz \\ Rechbauerstraße 12/II, A-8010 Graz \\ Projektleiter: Univ.-Prof. Dr.techn. Werner GOBIET \\ Projektpartner: \\ INSTITUT FÜR ZIVILRECHT (IZR) \\ Universität Innsbruck \\ HERRY CONSULT GMBH \\ Em. Univ.-Prof. Dr. Peter FALLER \\ VERFASSER: FORSCHUNGSGRUPPE AIp-EmiV \\ Univ.-Prof.Dr.techn. Werner GOBIET (ISV, Projektleiter) \\ Em.Univ.-Prof.Dr. Peter FALLER \\ Dr.techn. Markus FREWEIN (ISV, Projektmanagement) \\ Ass.-Prof. Mag. Dr. Peter JORDAN (IZR) \\ Dr. Max HERRY (Herry Consult Gmbh) \\ unter Mitarbeit von \\ Martin VILHAR (ISV) \\ ISBN-10 3-7001-3778-8 \\ ISBN-13 978-3-7001-3778-8 \\ doi: 10.1553/alp-emiv \\ http://epub.oeaw.ac.at/alp-emiv
}

Graz, im Juni 2006 


\section{Problemstellung und Ansatz}

\subsection{Warum sollte etwas geschehen?}

Seit den 1990er Jahren wird die Ökologisierung der Verkehrspolitik forciert. Der Umweltschutz ist ein bestimmendes Prinzip der Verkehrspolitik geworden. Die Schwerpunkte und instrumentellen Ansatzpunkte können in der verkehrspolitischen Ausrichtung unterschiedlich sein, und zwar

- Verbesserung der Verkehrsverhältnisse durch Verkehrswegeinvestitionen und Integration der Verkehrssysteme.

- Restriktive Gestaltung des Verkehrswesens. Es wird versucht die Verkehrsprobleme durch Verkehrsplanung und Bewirtschaftung zu lösen.

- Betonung des Umweltschutzzieles. Eine leistungsfähige Infrastruktur, effizientere Operationen in den Verkehrsabläufen durch Harmonisierung und Marktliberalisierung und die Anlastung der externen Kosten $^{11}$ sollen unterstützend wirken.

- Forcierung des „Nachhaltig ${ }^{12}$ Umweltverträglichen Verkehrs“. Ein Verkehrssystem ist nachhaltig umweltverträglich, wenn

- allgemein anerkannte Ziele im Hinblick auf die Gesundheit und Umweltqualität (z.B. die Ziele der WHO für die Schadstoff- und Lärmbelastung) eingehalten werden,

- die Integrität des Ökosystems nicht ernstlich in Frage gestellt ist und

- $\quad$ potenziell negative weltweite Phänomene, wie z.B. Klimaänderungen und die Zerstörung der stratosphärischen Ozonschicht sich tendenziell nicht verschlimmern.

- Die Abdeckung der grundlegenden Zugangserfordernisse und die Entwicklung von Einzelpersonen, Firmen und Organisationen in sicherer und der menschlichen Gesundheit sowie dem Ökosystem nicht abträglicher Weise ermöglicht und die Gerechtigkeit innerhalb aufeinander folgender Generationen und zwischen ihnen fördert,

\footnotetext{
${ }^{11}$ Unter externen Kosten versteht man Kosten die, z.B. durch Umweltverschmutzung (Produktionsausfälle, Waldschäden, Krankheit von Menschen) entstehen, aber nicht dem Verursacher, sondern anderen, z.B. der Allgemeinheit angelastet werden. Neueste Ansätze, auch in der Verkehrspolitik, zielen darauf ab, dass die so entstehenden Kosten dem Verursacher in Rechnung gestellt werden; man spricht dann von der „Internalisierung der externen Kosten“ (Prinzip der Kostenwahrheit).

${ }^{12}$ Es gibt einige Definitionen für „nachhaltig bzw. Nachhaltigkeit“. Die gängigste ist: Ein Konzept ist dann nachhaltig, wenn es ökologisch, ökonomisch und sozial verträglich ist und dadurch die Lebensgrundlage der nächsten Generationen sichert.
} 
- bezahlbar ist, effizient funktioniert, die Wahl des Verkehrsträgers ermöglicht, eine wettbewerbsfähige Wirtschaft und eine ausgewogenen Regionalentwicklung unterstützt,

- das Aufkommen an Emissionen und Abfallstoffen auf ein Maß beschränkt, das für das Ökosystem tragbar ist, erneuerbare Ressourcen maximal in dem Umfang nutzt, in dem diese erzeugt werden, nicht erneuerbare Ressourcen maximal in dem Umfang nutzt, in dem erneuerbare Ersatzstoffe entwickelt werden sowie Flächenverbrauch und Lärmentwicklung auf ein Minimum reduziert.

Es muss der Grundsatz der Verhältnismäßigkeit eingehalten werden, da prinzipiell der freie Dienstleistungs- und Warenverkehr unterbunden wird ${ }^{13}$.

Die Alpen sind durch das existierende Verkehrssystem und die Emissionsbelastungen des Straßengüterverkehrs massiv gefährdet. Die Immissionskonzentrationen von z.B. NO2 liegen deutlich über den erlaubten Grenzwerten, trotz der stetigen Verbesserung der Fahrzeugtechnologien ist durch den kontinuierlichen Zuwachs des Verkehrsaufkommens in vielen dieser Gebiete mit einem weiteren Anstieg der Schadstoffbelastung zu rechnen. In Österreich führen hochrangige Straßenverbindungen durch die Alpen und der Steuerungsmechanismus der Ökopunkteregelegung (1992 bis 2004) ist mittlerweile ausgelaufen. Der Ansatz dieses Steuerungsmechanismus war ökologisch motiviert, wenngleich Ergänzungen wünschenswert gewesen wären, die erwünschte Wirkung ist zwar eingetreten, eine Einhaltung der normativ verankerten Immissionskonzentrationen konnte im Vergleich zur technischen Verbesserung des eingesetzten Fahrzeugen nicht erreicht werden. Nach dem Auslaufen des Ökopunkteregimes existiert derzeit kein wirksames Verfahren zur ökologisch basierten Verkehrssteuerung in ökologisch sensiblen Gebieten ${ }^{14}$. Der Problemdruck ist demnach enorm und es ist notwendig problemadäquate Maßnahmen zu entwickeln: Einen Ansatz stellt das Verkehrslenkungsinstrument Alp-EmiV dar (vgl. Abbildung 4).

\footnotetext{
${ }^{13}$ Eventuell gelingt sogar der Nachweis, dass das Modell des Emissionszertifikatehandels im Straßengüterverkehr die am wenigsten beschränkende Maßnahme darstellt, dann könnte dieser bedenkenlos als EU-Rechts konform umgesetzt werden.

${ }^{14}$ Das IG-Luft erlaubt zwar verkehrsbeeinflussende Maßnahmen zur Verringerung der verkehrlichen Immissionsbelastungen ( $\mathrm{vgl}$. $\S \S 14,22)$ wie die Reduktion der Transporterfordernisse und zeitliche und räumliche Beschränkungen, die Wirksamkeit in Bezug auf die Immissionsbelastung wird aber nicht evaluiert (vgl. dazu auch Frewein 2005, S. 55 u. 62).
} 


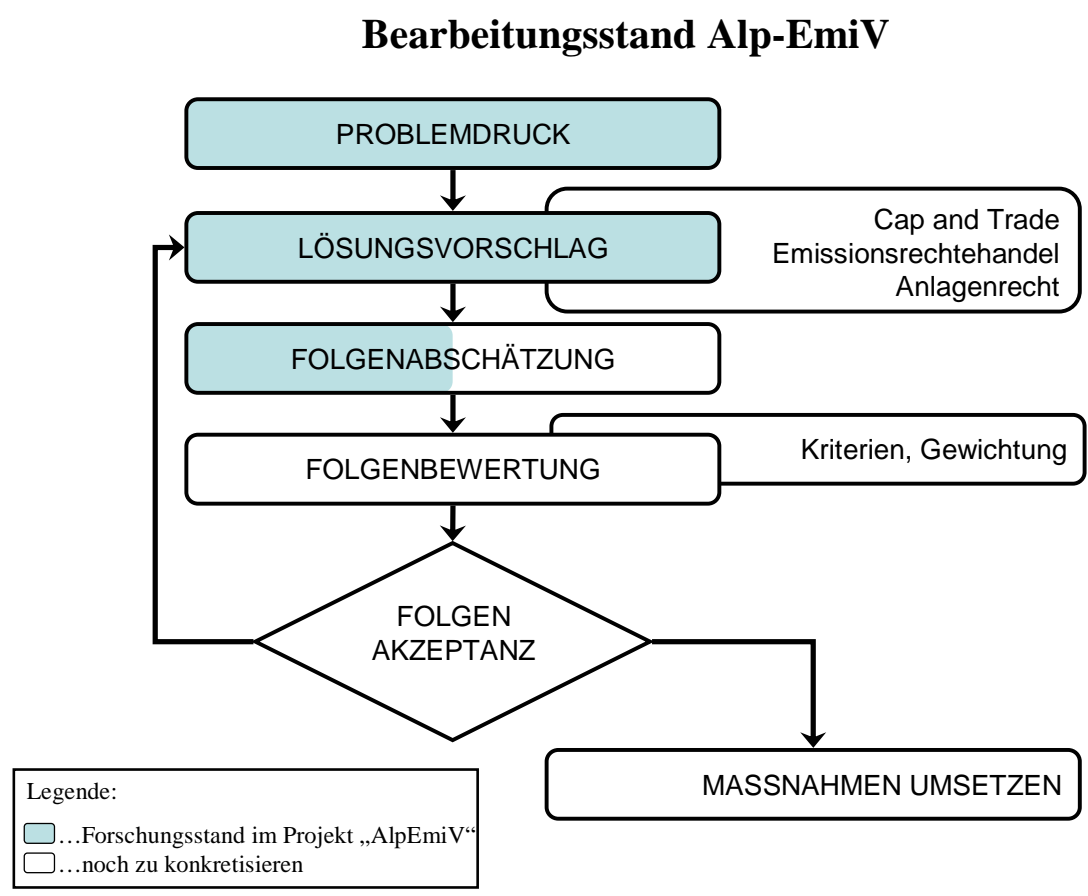

Abbildung 4: Bearbeitungsstand Alp-EmiV im gesamten Planungsprozess des Forschungsprojektes

\subsection{Forschungsfragen (-themen)}

Als Forschungsfragen werden die Fragen bezeichnet, die sich im Rahmen einer Forschungsaufgabe ergeben, respektive beantwortet werden. Für das gegenständliche Projekt ergibt sich durch den interdisziplinären Zugang eine Reihe von Fragen, die weit über den Untersuchungsgegenstand hinausgehen und teilweise anderen Fachdisziplinen zuzuordnen sind.

Für das gegenständliche Projekt stellt es sich demnach als zweckmäßig heraus, die gesammelten Probleme in Forschungsthemen zusammenzufassen. Aus der Vielzahl der Forschungsthemen ist ersichtlich, welchen Forschungsbedarf es im Bereich des „zukunftsfähigen und ökologisch verträglichen“ Verkehrs in ökologisch sensiblen Gebieten gibt. Die Forschungsthemen sind im Anhang angeführt.

Die Forschungsthemen wurden, in Übereinstimmung mit den Forschungsschwerpunkten, die von dem „Internationalen Wissenschaftlichen Komitee Alpenforschung" (ISCAR) mit dem Hintergrund einer alpenweiten, fachübergreifenden Forschungskooperation definiert wurden, wie folgt gegliedert:

- Allgemeine grundlegende Forschungsthemen,

- Gebietsbezogene Forschungsthemen,

- Infrastruktur- und verkehrsbezogene Forschungsthemen und 
- Das Lenkungsinstrument betreffende Forschungsthemen, die in

- Entwicklungsbezogene Forschungsthemen und

- Umsetzungsbezogene Forschungsthemen unterteilt werden.

\subsection{Ansatz Alp-EmiV}

\subsubsection{Das Prinzip von Alp-EmiV}

Das Prinzip von Alp-EmiV beruht auf der Idee, Autobahnabschnitte speziell in ökologisch sensiblen Gebieten wie Alpentälern, wo Emissionen auf Grund der Topographie eine besonders schädigende Wirkung haben, ähnlich wie Industrieanlagen einzustufen und in diesem Sinne von „Immissionsbegrenzten Europäischen Autobahnzonen" zu sprechen.

Ein wesentlicher Vorteil dieser Betrachtungsweise ist zweifelsohne, dass damit ein Abschnitt einer Straße betrachtet wird, und nicht die Emissionen eines Fahrzeuges. Weiters ergibt sich die Möglichkeit, zwischen verschiedenen Tageszeiten zu unterscheiden und auf ökologisch bedenkliche Witterungsbedingungen, wie zum Beispiel Inversionswetterlage oder Nebel, Rücksicht zu nehmen. Gerade in ökologisch sensiblen Gebieten wie dem Alpenraum ergibt sich durch diese Betrachtungsweise die Möglichkeit, eine Obergrenze für Emissionen festzulegen (ein so genanntes "Cap" zu definieren) und in weiterer Folge einen Handel mit Verschmutzungsrechten (Emissionsrechten) aufzubauen - das „Cap and Trade“ - System kann also im Straßenverkehr angewandt werden.

\subsubsection{Identifikation der wesentlichen Systemkomponenten}

Das Grundmodell enthält fünf Komponenten, welche in Tabelle 3 zusammengefasst sind:

- Käufer,

- Raum,

- Handelszeit,

- Nutzungszeit und

- Allokation.

- Beim Käufer der Zertifikate sind zwei Alternativen, wer am Handelssystem teilnehmen darf, zu klären:

- Jeder oder ein ausgewählter Personenkreis, wie z.B. Frächter o.ä..

- Bei der räumlichen Abgrenzung sind vier Möglichkeiten als Handelsraum für die Zertifikate gegeben:

- Lokale Einschränkung auf einzelne Alpenübergänge. 
- Nationale Einschränkung auf z.B. die österreichischen Alpenübergänge.

- Internationale Einschränkung auf alle Alpenübergänge innerhalb des Alpenbogens.

- Kontinentale Einschränkung auf alle definierten ökologisch sensiblen Gebiete innerhalb der EU (oder Europas)

- Die Handelszeit bezeichnet die Zeitspanne, in der ein Zertifikat am Sekundärmarkt gehandelt werden kann. Es ist zu unterscheiden zwischen

- befristet und

- unbefristet.

Ein unbefristet handelbares Zertifikat kann beispielsweise über die gesamte Handelsperiode, also verglichen mit den derzeit gültigen Emissionsrechtehandelsmodellen in der Industrie, über drei Jahre ge- und verkauft werden. Darüber hinaus kann auch die Mitnahme in die anschließende Periode gestattet werden. Für ein befristet handelbares Zertifikat sind in diesem Sinne entsprechende Zeitspannen durch die Umweltbehörde festzulegen.

- Zur Handelszeit ist auch die Nutzungszeit von wesentlicher Bedeutung, unterschieden wird zwischen

- Tageszeit und

- Jahreszeit.

Wobei jeweils zu unterscheiden ist, ob die Zertifikate befristet oder unbefristet gültig sein sollen (Stichwort: Slot-Management).

- Die Ausgabeform für die Zertifikate wird unter Allokation zusammengefasst. Es ist zu unterscheiden:

- Gratisvergabe (vgl. Faller,,,.,.)

- Versteigerung der Zertifikate und

- Verkauf zu einem fixen Preis.

Tabelle 3: Komponenten für das Emissionsrechtehandelssystem im Straßengüterverkehr (BADUT-System ${ }^{15}$ )

\begin{tabular}{|l|l|l|l|l|}
\hline \multicolumn{1}{|c|}{ KÄUFER } & \multicolumn{1}{|c|}{ RAUM } & \multicolumn{1}{c|}{ HANDELSZEIT } & NUTZUNGSZEIT & \multicolumn{1}{c|}{ ALLOKATION } \\
\hline \multicolumn{1}{|c}{ B... Buyer } & \multicolumn{1}{|c}{ A... Area } & T...Trading Period & \multicolumn{1}{c|}{ U... Usage Period } & D... Distribution \\
\hline Jeder & $\begin{array}{l}\text { Ein Alpenüber- } \\
\text { gang }\end{array}$ & Befristet & Tageszeit & Gratis \\
\hline $\begin{array}{l}\text { Ausgewählter } \\
\text { Personenkreis }\end{array}$ & $\begin{array}{l}\text { Alpenübergänge } \\
\text { eines Landes }\end{array}$ & unbefristet & Jahreszeit & Auktion \\
\hline & Alpenbogen & & & Fixpreis \\
\hline & $\begin{array}{l}\text { Ökologisch sen- } \\
\text { sible Gebiete der } \\
\text { EU }\end{array}$ & & & \\
\hline
\end{tabular}

${ }^{15}$ BADUT steht für die Anfangsbuchstaben der Komponenten des Emissionsrechtehandelssystems 
Abbildung 5 macht deutlich, in welch komplexer Weise diese Systemkomponenten mit den Fachbereichen interagieren.

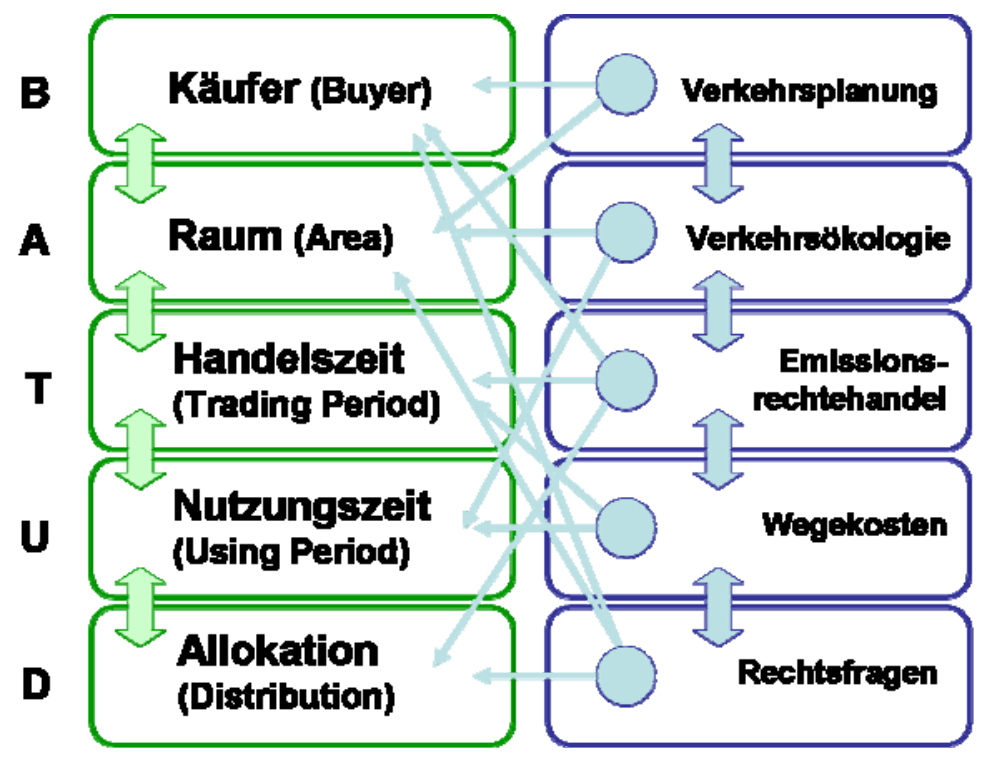

Abbildung 5: Interaktion zwischen Fachbereichen und Systembausteinen

\subsubsection{Resümee}

Durch das Prinzip das Cap so zu gestalten, dass es ökologisch hinterlegt ist und auf Emissionen beruht, kann gewährleistet werden, dass ein Anreiz geschaffen wird, um emissionssparende Technologien zu entwickeln und einzusetzen. Im Detail sind dazu noch folgende Fragen zu klären:

- Für welche Indikatoren wird das System ausgelegt? Gibt es einen Leitindikator oder müssen mehrere Indikatoren gleichberechtigt berücksichtigt oder speziell gewichtet werden?

- Die ausgegebenen Emissionsrechte (Durchfahrtsrechte) müssen in einer festen Einheit gehalten sein, wobei ein dynamischer Umrechnungsfaktor zwischen Emissionen und Immissionen festzusetzen ist, der aktuelle Witterungseinflüsse berücksichtigt.

- Die Frage der Vorausbestellung beziehungsweise Rückgabe von Emissionsrechten zusammen mit den gewährten zeitlichen Fristen ist festzulegen. 


\subsection{Einordnung des Ansatzes Alp-Emiv}

\subsubsection{Der Schweizer Ansatz - Vom Tropfenzähler zur Alpentransit- börse}

In der Schweiz wurde das sicherheitsbedingte Tropfenzählersystem auf der Gotthardroute Ende September 2002 mit der Wiedereinführung des Gegenverkehrs im Gotthardtunnel in Betrieb genommen und löste das Kreuzungsverbot für Lastwagen im Gotthardtunnel ab. Eine Weiterentwicklung davon ist das Reservationssystem, mit Hilfe dessen sich Transporteure einen Zeitabschnitt für eine Durchfahrt reservieren lassen können, während Fahrzeuge ohne Reservierung auf „Stand-By" stehen und weiterfahren können, sobald Durchfahrtskapazitäten frei sind.

Ein unterschiedlicher Lenkungsansatz für die Steuerung des Straßengüterverkehrs wird durch die Alpentransitbörse vertreten. Dabei werden Durchfahrtsrechte nicht mehr kostenfrei nach dem Prinzip „First come - First Served“ vergeben, sondern die Allokation wird über den Preis gesteuert. Zwei unterschiedliche Prinzipien werden dabei in der Literatur diskutiert:

- ein obligatorisches System handelbarer Alpentransitrechte, genannt „Cap and Trade",

- ein freiwilliges System handelbarer Reservationsrechte, welches als "SlotManagement mit dynamischer Preisgestaltung" bezeichnet wird.

Mit den beiden Varianten sind unterschiedliche Ziele verbunden: Das Modell "Cap and Trade" ist auf die Erreichung eines Mengenziels ausgerichtet, während das "Slot-Management mit dynamischer Preisgestaltung" eine bessere Auslastung der knappen Straßenkapazitäten erreichen soll. Bei dem „Cap and Trade“ Modell wird auch von einer Plafonierung gesprochen, wobei für den Plafond oder das Cap die Einschränkung des alpenquerenden Straßengüterverkehrs in der Schweiz ab 2009 auf 650.000 Fahrten pro Jahr gemäß dem Schweizer Verkehrsverlagerungsgesetz angenommen werden kann.

\subsubsection{Down-, Mid- und Up-Stream-Ansatz}

In Deutschland hat das Umweltbundesamt (UBA) im Juli 2003 die Bearbeitung des Vorhabens „Emissionsrechtehandel im Verkehr - Entwicklung von Ansätzen für ein Emissionsrechtehandelssystem im Verkehr" in Auftrag gegeben. Dabei werden folgende drei Ansätze für ein mögliches Emissionsrechtehandelssystem genannt:

- Down-Stream-Ansatz: Das letzte Glied in der Energieflusskette, der Endverbraucher der Treibstoffe, wird hierbei in die Pflicht genommen.

- Mid-Stream-Ansatz:

- Am Transportmittelhersteller: über eine Änderung der relativen Preise zwischen unterschiedlichen Fahrzeugtypen 
- Am Verkehrsdienstleister: zielt auf die Erfassung der Emissionen des öffentlichen Personenverkehrs und des Speditionsgüterverkehrs

- Up-Stream-Ansatz: Setzt bei der Bereitstellung von Kraftstoffen an.

Die Studie des Umweltbundesamts stellt die praktischen Umsetzungs- und Ausgestaltungsoptionen eines Up-Stream-Ansatzes sowie die damit unmittelbar einhergehenden Probleme einer Systemeinführung in den Vordergrund.

\subsubsection{Ausprägungsübersicht in Form eines Morphologischen Kas- tens}

Zur Analyse einer Problemstellung kann ein morphologischer Kasten angewandt werden, wobei das Problem zunächst in seine Bestandteile oder Merkmale zerlegt wird. Anschließend werden für jedes Merkmal die möglichen Ausprägungen festgelegt und Lösungsalternativen, die sich durch die Kombination von einzelnen Merkmalen ergeben, werden analysiert.

Tabelle 4 zeigt den morphologischen Kasten, wobei Merkmale und Ausprägungen auf die Darstellungen in der Alpentransitbörse (Ecoplan et al. 2004) sowie in dem im Rahmen dieses Berichts gezeigten BATUD-Modells (vgl. Tabelle 3). Die einzelnen Ausprägungen werden in Tabelle 5 genauer beschrieben. 
Tabelle 4: Morphologischer Kasten

\begin{tabular}{|c|c|c|c|c|c|c|c|}
\hline \multicolumn{3}{|c|}{ Merkmale } & \multicolumn{5}{|c|}{ Ausprägung } \\
\hline \multirow{6}{*}{ 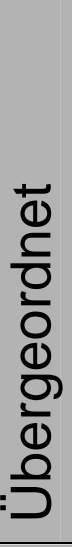 } & \multicolumn{2}{|c|}{$\begin{array}{l}\text { 1. Produkt (Durchfahrts- } \\
\text { recht) }\end{array}$} & 1. Einzelfahrt & \multicolumn{3}{|c|}{ 2. Emissionsmenge } & $\begin{array}{l}\text { 3. Räumlicher } \\
\text { Abschnitt }\end{array}$ \\
\hline & \multicolumn{2}{|c|}{$\begin{array}{l}\text { 2. Anreiz zur techno- } \\
\text { logschen Innovation }\end{array}$} & \multicolumn{2}{|c|}{ 1. Gegeben } & \multicolumn{3}{|c|}{ 2. Nicht gegeben } \\
\hline & \multicolumn{2}{|l|}{ 3. Grundprinzip } & 1. Plafonierung & \multicolumn{3}{|c|}{ 2. Dosierung } & $\begin{array}{l}\text { 3. Indikatorba- } \\
\text { sierende Steu- } \\
\text { erung }\end{array}$ \\
\hline & \multicolumn{2}{|c|}{$\begin{array}{l}\text { 4. Bindung des Durch- } \\
\text { fahrtsrechts }\end{array}$} & $\begin{array}{l}\text { 1. Fahrzeug } \\
\text { gebunden }\end{array}$ & \multicolumn{3}{|c|}{ 2. Kategoriespezifisch } & 3. Frei \\
\hline & \multicolumn{2}{|c|}{ 5. Durchfahrtsgarantie } & 1. Keine & \multicolumn{3}{|c|}{ 2. Gleicher Tag } & 3. Slot \\
\hline & \multicolumn{2}{|c|}{ 6. Plattform für Handel } & 1. Keine & \multicolumn{3}{|c|}{ 2. Betreiber } & 3. Warenbörse \\
\hline \multirow{4}{*}{$\mathrm{B}$} & \multicolumn{2}{|l|}{ 7. B...Käufer } & \multicolumn{4}{|c|}{ 1. Jeder } & r Personenkreis \\
\hline & \multicolumn{2}{|l|}{ 8. Teilnahme } & \multicolumn{2}{|c|}{ 1. Obligatorisch } & \multicolumn{3}{|c|}{ 2. Freiwillig } \\
\hline & \multicolumn{2}{|c|}{ 9. Recht zum Ersterwerb } & \multicolumn{2}{|c|}{ 1. Alle } & \multicolumn{3}{|c|}{ 2. Lizenzierte Teilnehmer } \\
\hline & \multicolumn{2}{|c|}{ 10. Teilnahme am Handel } & 1. Kein Handel & \multicolumn{3}{|c|}{ 2. Lizenzierte Teilnehmer } & 3. Alle \\
\hline \multirow{3}{*}{$\mathrm{A}$} & \multicolumn{2}{|l|}{ 11. A...Raum } & $\begin{array}{c}\text { 1. Einzelner } \\
\text { Alpenübergang }\end{array}$ & \multicolumn{3}{|c|}{$\begin{array}{c}\text { 2. Alpenübergänge eines } \\
\text { Landes }\end{array}$} & 3. Alpenbogen \\
\hline & \multicolumn{2}{|c|}{ 12. Kurzstreckenverkehr } & \multicolumn{3}{|c|}{ 1. Sonderbehandlung } & \multicolumn{2}{|c|}{ 2. Keine Sonderbehandlung } \\
\hline & 13. Grundprinzip & & 1. Plafonierung & & Dosier & Ing & $\begin{array}{l}\text { 3. Indikatorba- } \\
\text { sierende Steu- } \\
\text { erung }\end{array}$ \\
\hline & 14. T...Handelsze & & 1. Begr & & & 2. Ur & egrenzt \\
\hline $\mathrm{T}$ & $\begin{array}{l}\text { 15. Häufigkeit de } \\
\text { kation }\end{array}$ & Erstallo- & 1. Einmalig & & Mehrm & alig & $\begin{array}{l}\text { 3. Kontinuier- } \\
\text { lich }\end{array}$ \\
\hline & 16. Annulationsm & lichkeit & 1. Keine & 2. Mit & ntsch & idigung & 3. Versicherbar \\
\hline & $17 . / 18$ & Tag & 1. BegI & & & 2. Ur & egrenzt \\
\hline & U...Nutzungszeit & Jahr & 1. BegI & & & 2. Ur & egrenzt \\
\hline & 19. Slotlänge & & 1. Jahr & 2. Monat & $\begin{array}{c}3 . \\
\mathrm{Tag}\end{array}$ & $\begin{array}{l}\text { 4. Halb- } \\
\text { tag }\end{array}$ & 5. Stunde(n) \\
\hline & 20. Erworbenes $\mathrm{F}$ & & 1. Durchfahrt & 2. $s$ & tbeni & zung & 3. Überholrecht \\
\hline & $\begin{array}{l}\text { 21. Reservationsr } \\
\text { keit }\end{array}$ & glich- & 1. $\mathrm{J}$ & & & & Jein \\
\hline & 22. D...Allokation & & 1. Gratis & & Verk & & 3. Auktion \\
\hline $\mathrm{D}$ & 23. Art der Erstall & ation & 1. Gratis & & Verk & & 3. Auktion \\
\hline & $\begin{array}{l}\text { 24. Zeitpunkt de } \\
\text { kation }\end{array}$ & Erstallo- & $\begin{array}{l}\text { 1. Monate im } \\
\text { Voraus }\end{array}$ & 2. $\mathrm{T}$ & e im & oraus & 3. Sofort \\
\hline
\end{tabular}

BATUD - Überlegung Überlegungen in Übereinstimmung mit den ECOPLAN/Rapp Trans

Ökologische Empfehlung

FORSCHUNGSGRUPPE ALP-EMIV: Gobiet, Faller, Frewein, Herry, Jordan

Seite 36 
Tabelle 5: Beschreibung der Merkmale und Ausprägungen

\begin{tabular}{|c|c|}
\hline Merkmal & Erläuterung \\
\hline Produkt (Durchfahrtsrecht) & $\begin{array}{l}\text { Beschreibt jenes Recht, welches mit dem Erwerb eines Zertifi- } \\
\text { kats gewährt wird. Dabei kann es sich um das Zurücklegen } \\
\text { einer definierten Strecke oder die Emission einer bestimmten } \\
\text { Schadstoffmenge handeln. }\end{array}$ \\
\hline $\begin{array}{l}\text { Anreiz zur technologischen Inno- } \\
\text { vation }\end{array}$ & $\begin{array}{l}\text { Definiert, ob durch die Auslegung des Systems ein Anreiz } \\
\text { gegeben ist, in technologische Innovationen zu investieren } \\
\text { anstatt Zertifikate einzulösen, womit die ökologische Ausrich- } \\
\text { tung des Systems ausgedrückt wird. }\end{array}$ \\
\hline Grundprinzip & $\begin{array}{l}\text { Durch eine Plafonierung wird die Anzahl der Fahrten begrenzt, } \\
\text { eine Dosierung meint die sicherheitsbedingte Limitierung, z.B. } \\
\text { ein Tropfenzähler à la Gotthardtunnel. Mittels einer Indikator } \\
\text { basierenden Steuerung kann eine dynamische Einflussnahme, } \\
\text { beispielsweise entsprechend aktueller Emissions-Immissions- } \\
\text { Parameter, erreicht werden. }\end{array}$ \\
\hline Bindung des Durchfahrtsrechts & $\begin{array}{l}\text { Beschreibt die mögliche Bindung an ein Fahrzeug oder an } \\
\text { eine Fahrzeugkategorie. }\end{array}$ \\
\hline Durchfahrtsgarantie & $\begin{array}{l}\text { Beschreibt, ob für den Inhaber eines Zertifikats die Durchfahrt } \\
\text { garantiert wird. }\end{array}$ \\
\hline Plattform für Handel & $\begin{array}{l}\text { Der Handel der Zertifikate kann direkt zwischen Transporteu- } \\
\text { ren, über eine eigene Plattform oder eine bestehende Einrich- } \\
\text { tung (z.B. Warenbörse) abgewickelt werden. }\end{array}$ \\
\hline B...Käufer & $\begin{array}{l}\text { Es kann entweder jedem, oder einem ausgewählten Kreis } \\
\text { (z.B. Transporteuren) gewährt werden, Zertifikate zu kaufen } \\
\text { und damit am Handel teilzunehmen. }\end{array}$ \\
\hline Teilnahme & $\begin{array}{l}\text { Das System kann auf freiwilliger Basis oder verpflichtend } \\
\text { abgewickelt werden. }\end{array}$ \\
\hline Recht zum Ersterwerb & $\begin{array}{l}\text { Ähnlich wie bei Komponente „B...Käufer“ kann jeder oder nur } \\
\text { lizenzierte Teilnehmer in das System einbezogen werden. }\end{array}$ \\
\hline Teilnahme am Handel & $\begin{array}{l}\text { Die Handelsmöglichkeit kann für alle oder für einen be- } \\
\text { schränkten Kreis bestehen. Alternativ könnte auch kein Han- } \\
\text { del stattfinden. }\end{array}$ \\
\hline A...Raum & $\begin{array}{l}\text { Die räumliche Ausdehnung des Systems kann für einen Al- } \\
\text { penübergang, die Alpenübergänge eines Landes oder den } \\
\text { gesamten Alpenbogen bestehen. }\end{array}$ \\
\hline Kurzstreckenverkehr & $\begin{array}{l}\text { Für den Kurzstreckenverkehr kann eine Priorität (uneinge- } \\
\text { schränktes Durchfahrtsrecht) vorgesehen werden. }\end{array}$ \\
\hline Grundprinzip & $\begin{array}{l}\text { Durch eine Plafonierung wird die Anzahl der Fahrten begrenzt, } \\
\text { eine Dosierung meint die sicherheitsbedingte Limitierung, z.B. } \\
\text { ein Tropfenzähler à la Gotthardtunnel. Mittels einer Indikator } \\
\text { basierenden Steuerung kann eine dynamische Einflussnahme, } \\
\text { beispielsweise entsprechend aktueller Emissions-Immissions- } \\
\text { Parameter, erreicht werden. }\end{array}$ \\
\hline T...Handelszeit & $\begin{array}{l}\text { Die Handelsmöglichkeit eines Zertifikats innerhalb eines Allo- } \\
\text { kationszeitraums kann unbegrenzt oder eingeschränkt ge- } \\
\text { währt werden. }\end{array}$ \\
\hline Häufigkeit der Erstallokation & $\begin{array}{l}\text { Bestimmt, wie oft Emissionszertifikate ausgegeben werden. } \\
\text { Als Bezugszeitraum gilt eine spezifisch definierte Allokations- } \\
\text { periode. }\end{array}$ \\
\hline Annulationsmöglichkeit & $\begin{array}{l}\text { Beschreibt, ob und mit welcher Form von Entschädigung ein } \\
\text { Zertifikat nach der Einlösung (Erklärung der Gültigkeit) zu- } \\
\text { rückgegeben werden kann. }\end{array}$ \\
\hline U...Nutzungszeit & $\begin{array}{l}\text { Das Einlösen eines erworbenen Zertifikats, sowohl über den } \\
\text { Tag als auch über das Jahr kann beschränkt oder unbe- } \\
\text { schränkt sein. }\end{array}$ \\
\hline Slotlänge & $\begin{array}{l}\text { Beschreibt die Länge des Zeitraums, der für die Durchfahrt } \\
\text { gewährt wird. }\end{array}$ \\
\hline
\end{tabular}




\begin{tabular}{|l|l|}
\hline Erworbenes Recht & $\begin{array}{l}\text { Durch den Kauf eines Zertifikats kann entweder das Recht zur } \\
\text { zeitlich nicht festgelegten Durchfahrt für den definierten Raum, } \\
\text { die Durchfahrt zu einem bestimmten Zeitpunkt oder das Über- } \\
\text { holen wartender Fahrzeuge erworben werden. Dabei handelt } \\
\text { es sich um eine Besonderheit, angedacht im Rahmen der } \\
\text { Schweizer Alpentransitbörse, wobei die Konformität mit gülti- } \\
\text { gem EU-Recht zu überprüfen wäre. }\end{array}$ \\
\hline Reservationsmöglichkeit & $\begin{array}{l}\text { Für die Benutzung des definierten Raumes kann eine Reser- } \\
\text { vierung vorgenommen werden. }\end{array}$ \\
\hline D...Allokation & $\begin{array}{l}\text { Die Erstausgabe der Zertifikate kann entweder gratis, zu ei- } \\
\text { nem Fixpreis oder durch eine Auktion erfolgen. }\end{array}$ \\
\hline Art der Erstallokation & $\begin{array}{l}\text { Definiert, wie lange im Voraus ein Recht erworben werden } \\
\text { kann. }\end{array}$ \\
\hline Zeitpunkt der Erstallokation &
\end{tabular}

Der charakteristische Unterschied zwischen dem Schweizer Modell „Alpentransitbörse" und dem Alp-EmiV Modell ist die Ausrichtung des Caps. Bei der Alpentransitbörse erfolgt dies über ein definiertes Cap, welches im Verkehrsverlagerungsgesetz festgeschrieben ist. Im Unterschied dazu ist im Rahmen des Modells „AlpEmiV“ ein dynamisches Cap, das ökologisch hinterlegt ist, vorgesehen. Damit kann durch diesen Modellansatz eine lokale ökologische Wirksamkeit für den gewählten Raum erreicht werden. In Abbildung 6 ist das der Projektdesign von AlpEmiV schematisch dargestellt.

\section{Projektdesign}

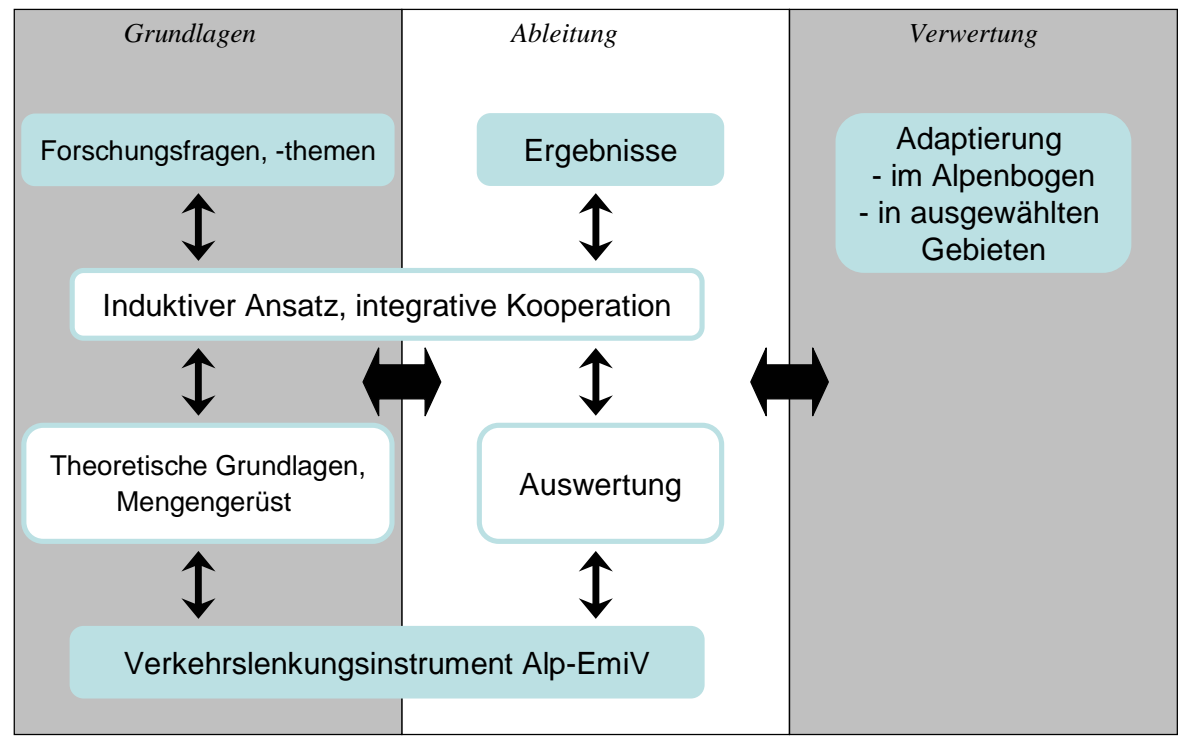

\section{Abbildung 6: Projektdesign AlpEmiV}

